\title{
Satellite remote sensing of tropospheric composition - principles, results, and challenges
}

\author{
A. Richter, \\ Institute of Environmental Physics, University of Bremen, Otto-Hahn-Allee 1, \\ 28359 Bremen, Germany
}

\begin{abstract}
Over the last years, satellite observations of tropospheric composition have become an important data source for atmospheric chemistry and air pollution applications. Most of these measurements are performed using passive remote sensing in the UV, visible and IR spectral regions. The choice of wavelengths used determines the species that can be retrieved but also the vertical sensitivity and resolution of the measurements. Here, a brief introduction into space-borne remote sensing of the troposphere is given, a few typical examples are discussed and an outlook to future developments is given.
\end{abstract}

\section{Introduction}

The troposphere is the lowest part of the atmosphere reaching from the surface up to the tropopause which is at 8 to $18 \mathrm{~km}$ altitude depending on latitude and season. In the troposphere, temperature usually decreases with altitude leading to vertical mixing and convection. It is characterised by weather phenomena such as clouds, rain and rapid horizontal and vertical transport. The troposphere is also the part of the atmosphere which is in direct contact to the biosphere including humans.

Most emissions into the atmosphere take place close to the ground. This includes direct anthropogenic emissions from e.g. energy production, transport or industrial activities, indirect anthropogenic emissions from agriculture, biomass burning or other land use, and natural emissions from the biosphere or surface. As a result, a large number of different trace gases and particles can be found in the air, where they can undergo photochemical reactions and participate in chemistry on and in droplets and aerosols until they are either converted into inert substances or are removed from the atmosphere by dry or wet deposition. Depending on their atmospheric lifetimes, emissions can be transported over different distances from local scales as in the case of short lived NOx to continental scales as for $\mathrm{CO}$ or globally as is the case for CFCs.

This complex interaction of emissions, chemistry and transport in the troposphere is of large scientific interest. At the same time, it is also highly relevant for both the environment and human health, as many of the substances emitted into the troposphere adversely affect human health and the biosphere. Well known examples are acid rain, summer ozone smog and the occurrence of high aerosol loadings in polluted cities. The emission of greenhouse gases is of similar importance although the effects on humans are indirect and long-term but possibly even more threatening than traditional pollution.

In order to describe tropospheric composition and to investigate emissions, chemical transformation and transport of key substances, accurate measurements are needed in polluted regions but also in remote areas. Many countries have operational air quality networks and long-term scientific measurement programmes but these are limited to certain areas. Intensive measurement campaigns involving

\footnotetext{
a e-mail: andreas.richter@iup.pysik.uni-bremen.de
}

This is an Open Access article distributed under the terms of the Creative Commons Attribution-Noncommercial License 3.0, which permits unrestricted use, distribution, and reproduction in any noncommercial medium, provided the original work is properly cited. 
aircraft and other platforms are used for detailed investigations of atmospheric processes during short episodes. These data can be complemented by satellite observations of the troposphere which provide the global picture for a number of substances albeit often with reduced accuracy for individual measurements.

In this article, a brief introduction is given in the principles of satellite remote sensing of the troposphere focusing on trace gases in the lower troposphere and not covering aerosols or meteorology observations. A few examples are given for measurement results from current satellite sensors followed by an outlook on future developments. A much more detailed discussion of satellite remote sensing of the troposphere can be found in [1], and more examples from different sensors are given in [1-3].

\section{Basic measurement principle and radiative transfer}

All satellite remote sensing measurements of the troposphere are based on the use of electromagnetic radiation and its interaction with constituents in the atmosphere. Most of the techniques used today apply passive remote sensing where the instrument does not have its own source of radiation but rather uses radiation from the sun scattered in the atmosphere or the thermal emission of the atmosphere itself. Active remote sensing methods such as RADAR, Lidar or radio occultation measurements will not be discussed here. Observations are mainly done in nadir geometry although occultation and limb observations can provide some information on the upper troposphere as well.

Within the passive remote sensing methods, the main distinction is between the different wavelength regions which all have their advantages and disadvantages depending on the quantity of interest. In all cases, atmospheric species are identified using the spectral dependence of their absorption or emission signals in the measurements, and their amounts are deduced from the observed absorption or emission strength by comparison to modelled values. While the details of the retrieval approaches taken vary strongly between applications, the basic principle is similar in all cases. A radiation transfer model is used to simulate the signal observed by the instrument using some a priori information on the atmospheric composition. The simulated and the measured signal are compared and depending on the level of (dis-)agreement, parameters such as the trace gas profile are changed and a new simulation is performed. This process is repeated iteratively until agreement is found within predefined limits. It also is a common characteristic of the satellite data analysis that not all necessary information can be retrieved from the measurements and that the results therefore depend on a priori assumptions made e.g. on the vertical distribution of a trace gas or on the surface emissivity.

Depending on the wavelength range used, the sensitivity to different layers in the troposphere varies which needs to be taken into account when interpreting the results. This is the result of the varying relevance of different processes in radiative transfer through the atmosphere as sketched in Fig. 1. In the following, the different wavelength regions relevant for remote sensing of tropospheric composition from satellite will briefly be discussed.

Some of the first remote sensing observations of the troposphere were based on the use of UV wavelengths in the TOMS instrument [4]. Both ozone and sulphur dioxide $\left(\mathrm{SO}_{2}\right)$ have strong absorption structures at wavelengths from $300-350 \mathrm{~nm}$, and these have been used to detect ozone in the stratosphere but also in the troposphere [5], as well as the emission of $\mathrm{SO}_{2}$ from volcanoes [6]. Using the GOME instrument which has better spectral resolution and signal to noise, even strong $\mathrm{SO}_{2}$ pollution sources could be detected [7]. Using somewhat longer wavelengths in the UV and the better spectral resolution of the GOME instrument, much weaker absorption signals can be detected from species such as $\mathrm{BrO}[8]$ and $\mathrm{HCHO}[9]$.

In the UV, thermal emission of the surface and the atmosphere can be neglected and only sun light needs to be considered. As Rayleigh scattering depends on wavelength with $\lambda^{-4}$, it is of large importance in the UV. Many photons are scattered before they reach the surface, and therefore the sensitivity of the satellite measurements to the lower troposphere is strongly reduced. This is reinforced by the fact that most surfaces have a low reflectivity of just a few percent at UV wavelengths with the exception of ice and snow. Over bright surfaces, multiple scattering enhances light path length and thereby leads to increased sensitivity of the measurements to the lowest atmospheric layers.

With the GOME, SCIAMACHY, OMI, and GOME-2 instruments, measurements at good spectral resolution $(0.2-0.4 \mathrm{~nm})$ in the visible part of the spectrum became available. In this spectral range, 


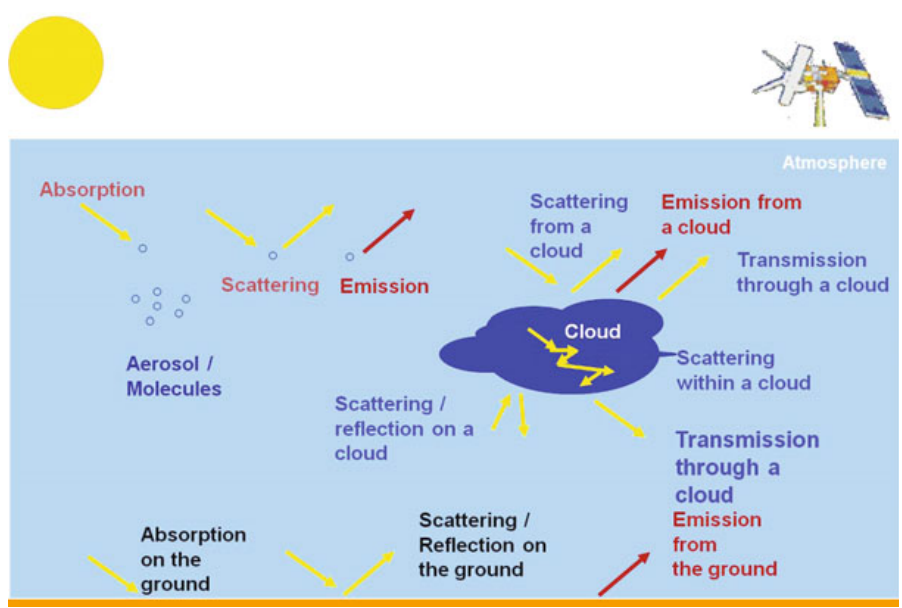

Fig. 1. Overview over the most important processes in radiative transport through the atmosphere.

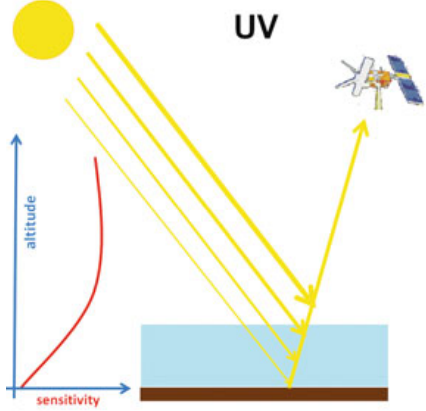

NIR

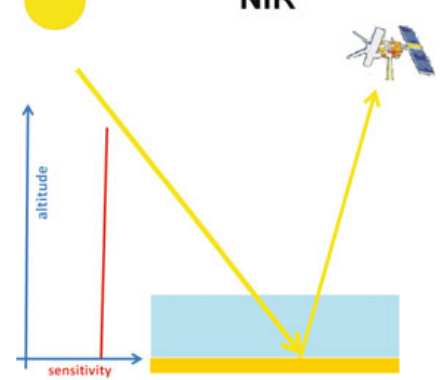

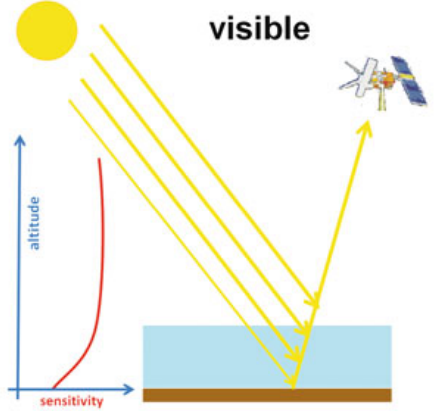

IR

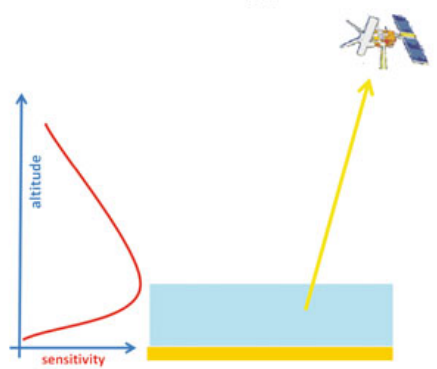

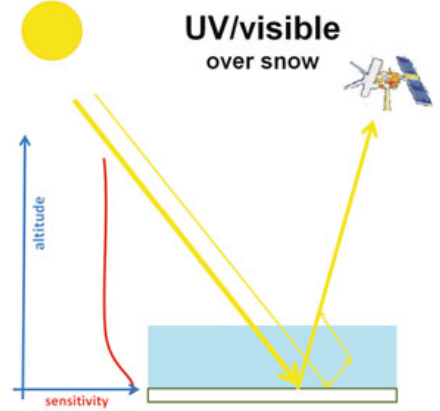

IR

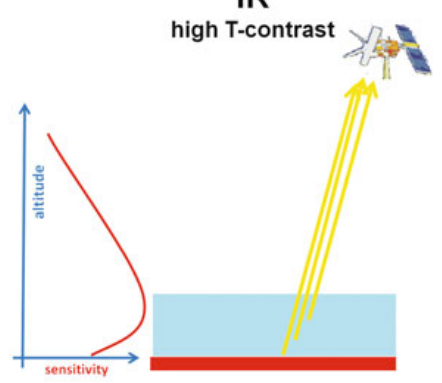

Fig. 2. Sketch of measurement geometry and vertical sensitivity for satellite observations of the troposphere in different wavelength ranges. See text for details.

additional trace gases such as $\mathrm{NO}_{2}$ [10], glyoxal [11] and IO [12] have absorption features and therefore can be detected. The solar intensity is larger than in the UV which improves the signal to noise ratio. Reduced Rayleigh scattering in combination with higher surface albedo increases measurement sensitivity to the lower troposphere but it is still lower than for the middle and upper troposphere. As in the case of UV observations, the largest signal from the boundary layer is obtained over snow and ice.

In the NIR spectral region, scattering is of little relevance and thermal emission is still weak. As a result, most of the photons detected by the satellite instrument have taken the path from the sun to the surface and back, providing nearly homogeneous sensitivity to all atmospheric layers. This ideal situation is found for $\mathrm{CO}, \mathrm{CH}_{4}$ and $\mathrm{CO}_{2}$ measurements by SCIAMACHY [13-15] and in the shortwave channel of MOPITT [16]. However, the absence of atmospheric scattering also implies that 
signals are small in particular over dark surfaces such as the oceans, limiting the signal to noise ratio of the measurements.

In the thermal IR ( $3 \mu \mathrm{m}-100 \mu \mathrm{m})$, a large number of different atmospheric species can be observed if spectral resolution is good enough to resolve the individual spectral lines. In contrast to the other spectral ranges, the light source is not the sun but thermal emission of the surface and the atmosphere itself, and signals are a combination of emission and absorption in the atmosphere. The vertical sensitivity depends on the temperature profile and the thermal contrast between surface and atmosphere and usually is highest in the middle troposphere with very low sensitivity to the surface layers. However, good thermal contrast between surface and atmosphere can lead to significant sensitivity also to the lowest layers. Nadir thermal IR measurements are performed by e.g. AIRS [17], TES [18] and IASI $[19,20]$ and in limb geometry also by MIPAS [21]. Measurements in the microwave part of the spectrum can also be used to detect the emissions of trace species, but application to tropospheric gases is limited by the very strong $\mathrm{H}_{2} \mathrm{O}$ signal which turns the atmosphere opaque at most frequencies.

\section{Some considerations for satellite observations}

\subsection{Vertical resolution}

Ideally, satellite observations should provide a height resolved measurement in the troposphere. For measurements in the thermal IR, the temperature dependence and also pressure broadening of the lines provides such information. Depending on the strength of the lines, the spectral resolution of the measurements, and the thermal contrast, $1-4$ independent pieces of information can be retrieved from the spectra. In the UV and visible part of the spectrum, the measurements contain very little information on the vertical distribution of the species, usually providing just one piece of information (the tropospheric column amount). In principle, observations at different wavelengths contain vertical information as the penetration depth of photons increases towards longer wavelengths, and for volcanic plumes of $\mathrm{SO}_{2}$, this has already been exploited [22]. Combination of UV, visible and IR measurements should add additional vertical resolution but only if radiative transfer and molecular parameters are fully consistent which is difficult.

\subsection{Spatial resolution}

The spatial resolution of current satellite instruments varies from $12 \mathrm{~km}$ diameter (IASI) to $80 \times$ $40 \mathrm{~km}^{2}$ (GOME-2). While this is good enough to map tropospheric concentration fields at the regional scale, it is not fine enough to resolve individual power plants or even large cities in detail. For those species having short atmospheric life times (e.g. $\mathrm{NO}_{2}$ ), the averaging over large satellite pixels leads to significant dilution of signals from point sources, complicating quantitative analysis and separation of emission sources. Also, variations in surface reflectivity or emissivity within one pixel lead to problems in the interpretation of the results in particular in coastal areas and close to mountains. An additional problem arises from clouds in the field of view, the probability of which strongly increases for large ground pixels.

\subsection{The effect of clouds}

In all wavelength regions discussed here, thick clouds are opaque and basically shield the atmosphere below the cloud from the satellite view. This leads to a significant reduction in the number of measurements that can be used for retrievals down to the surface. In the UV and visible, light path enhancement through multiple scattering increases the measurement sensitivity within the upper part of a cloud and also above a cloud, an effect that is of particular importance for the detection of lightning NOx emissions [23] but also for the interpretation of elevated pollution plumes e.g. from biomass burning. In case of partially cloudy pixels, corrections can be applied using cloud fraction and cloud top in combination with a priori assumptions but at the expense of increased uncertainties. 


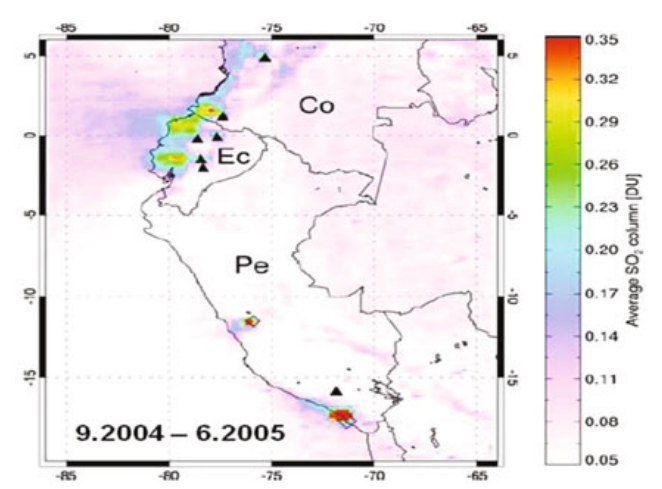

Fig. 3. OMI derived $\mathrm{SO}_{2}$ columns over Colombia, Ecuador and Peru. Triangles indicate volcanoes, diamonds are Peruvian copper smelters. Figure taken from [25].

A more subtle effect of the inability to measure tropospheric composition in cloudy situations is a clear sky bias in averages derived from satellite observations. Photochemistry, atmospheric transport and precipitation are all linked to weather conditions, and satellite observations only sample the clear sky part of these values.

\subsection{Temporal and spatial sampling}

Although the number of individual satellite measurements per day is very large, the statistics of clear sky observations over a given location is often poor even on a monthly basis. As a result, monthly or annual averages are often used for satellite data whereas surface observations usually provide high temporal resolution. If values from a larger area are averaged to improve the signal to noise ratio, spatial mismatch can become a problem too, e.g. if data around a power plant are averaged that include many measurements upwind of the plume.

Most of the current atmospheric chemistry instruments are on platforms having sun-synchronous orbits. As a result, they sample each location at a constant local time (plus a second measurement at night for thermal infrared observations). These measurements can therefore not resolve the diurnal variation of the tropospheric trace-gas concentrations. This effect has been shown to be important for $\mathrm{NO}_{2}$ by comparing results from the OMI and SCIAMACHY instruments which are on platforms having different overpass times [24].

\section{Some examples}

Over the last years, a large number of satellite data products for tropospheric species became available. Here, only a few examples are presented showing results obtained in the different wavelength regions from the UV to the thermal IR. A much more complete and relatively recent overview of satellite measured data can be found in [1].

\subsection{OMI Observations of $\mathrm{SO}_{2}$ pollution}

Sulphur dioxide has strong absorptions in the UV part of the spectrum which can be used to retrieve its atmospheric abundance. In the example shown in Fig. 3, data from the OMI instrument operating on Aura since August 2004 have been utilised. The OMI instrument is a two channel imaging UV/vis grating spectrometer providing daily coverage with good spatial resolution of $13 \times 24 \mathrm{~km}^{2}$ at sub satellite point. For the $\mathrm{SO}_{2}$ retrieval, a band residual difference algorithm (BR) has been applied to 4 wavelengths between 310.8 and $314.4 \mathrm{~nm}$ [25]. Although the sensitivity to the lower troposphere is 


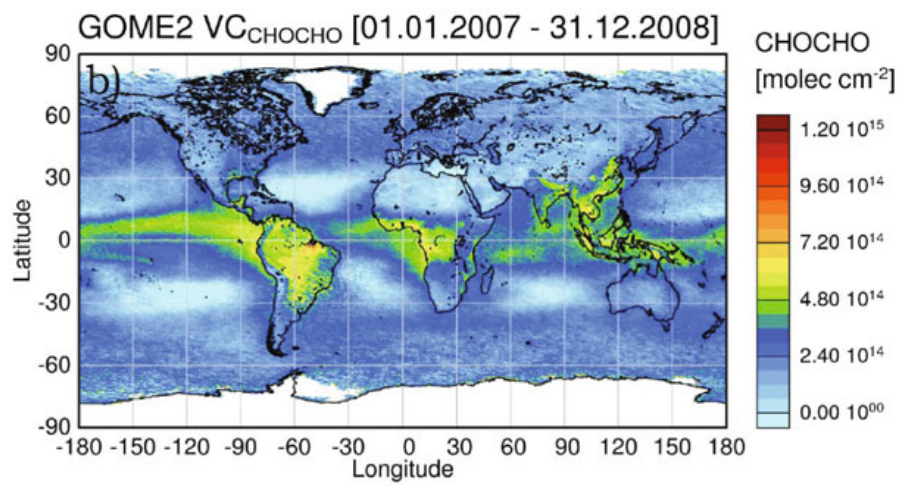

Fig. 4. Global glyoxal distribution as derived from GOME-2 measurements. Figure taken from [26].

small at these wavelengths, clear $\mathrm{SO}_{2}$ signals can be retrieved not only for the volcanoes in the region which emit $\mathrm{SO}_{2}$ to higher altitudes, but also for two large copper smelters in Peru (Ilo and La Oraya) where $\mathrm{SO}_{2}$ is expected to be very close to the ground. These smelters emitted large quantities of $\mathrm{SO}_{2}$ to the atmosphere, causing significant health and environmental problems. In subsequent years, $\mathrm{SO}_{2}$ emissions have reduced due to modernisation and reduction in production, highlighting the ability of OMI to monitor $\mathrm{SO}_{2}$ emissions and their changes from space, at least for large sources.

\subsection{GOME-2 measurements of glyoxal}

The GOME-2 instrument is a 4 channel UV/vis grating spectrometer launched onboard MetOp-A in October 2006. The ground pixel size of GOME-2 is $40 \times 80 \mathrm{~km}^{2}$, and with a swath width of $1920 \mathrm{~km}$, nearly global coverage is achieved every day. Glyoxal retrievals are performed in the blue part of the spectrum at 424-457 nm using the Differential Optical Absorption Spectroscopy (DOAS) method. Details on the retrieval can be found in [26].

Glyoxal (CHO.CHO) is one of the most prevalent VOCs in the troposphere. Glyoxal is produced in the atmosphere through the oxidation of precursor VOCs which can be either of biogenic origin, from anthropogenic emissions or from biomass burning, the relative importance of the different sources still being under investigation. As can be seen in Fig. 4, the largest glyoxal columns are found in the tropics over regions with biogenic emissions and biomass burning, but also downwind of the source regions. The latter is not reproduced by model simulations [27] but confirmed by ship-borne measurements [28]. Possible explanations are transport of an unknown biogenic precursor and also biogenic emissions from the ocean. An interesting application of the glyoxal measurements is comparison to simultaneous retrievals of formaldehyde (HCHO) [26]. The ratio of these two VOCs varies between anthropogenic, biogenic and pyrogenic sources, providing a way to classify VOC sources from satellite.

\subsection{SCIAMACHY retrievals of $\mathrm{CO}_{2}$}

The SCIAMACHY instrument launched on board of ENVISAT in March 2002 is an 8 channel grating spectrometer covering wavelengths from 240 to $2380 \mathrm{~nm}$. With its NIR channels, SCIAMACHY enables retrieval of methane $\left(\mathrm{CH}_{4}\right)$ and carbon dioxide $\left(\mathrm{CO}_{2}\right)$ columns down to the surface. Carbon dioxide retrievals are performed in the $1558-1594 \mathrm{~nm}$ range and are normalised by simultaneous $\mathrm{O}_{2}$ measurements at $755-775 \mathrm{~nm}$. Normalisation removes the strong dependence of the $\mathrm{CO}_{2}$ signal on total air column and makes the values comparable to mixing ratios as measured by in-situ instruments.

As shown in Fig. 5, SCIAMACHY observations show both the seasonal variation and the increase from year to year reported by in-situ measurements. In the northern hemisphere, summer $\mathrm{CO}_{2}$ levels are lower as result of increased plant growth. At the same time, anthropogenic emissions continue to 


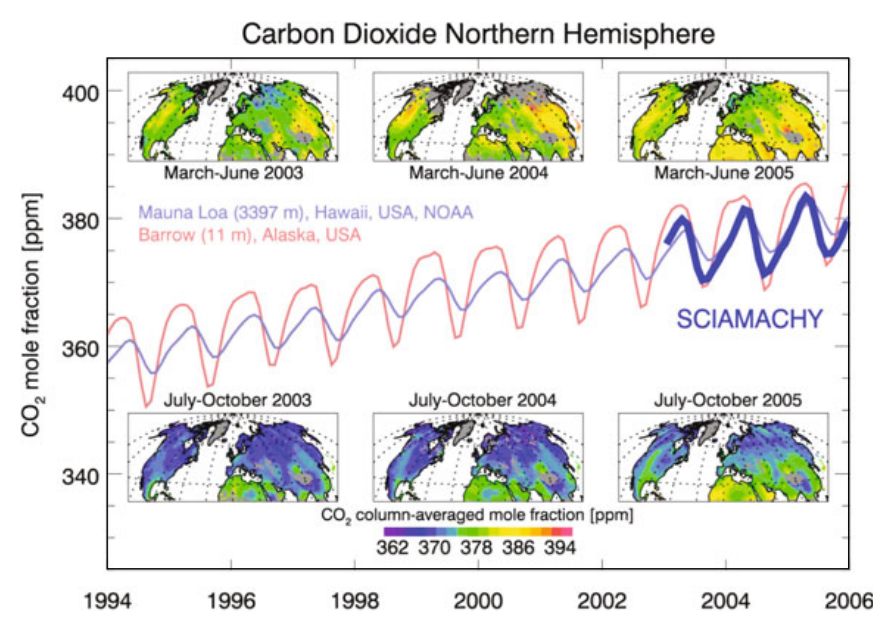

Fig. 5. SCIAMACHY column averaged mole fractions of $\mathrm{CO}_{2}$ in the northern hemisphere in different seasons and years, compared to in-situ observations in Mauna Loa and Barrow. Figure adapted from [14].

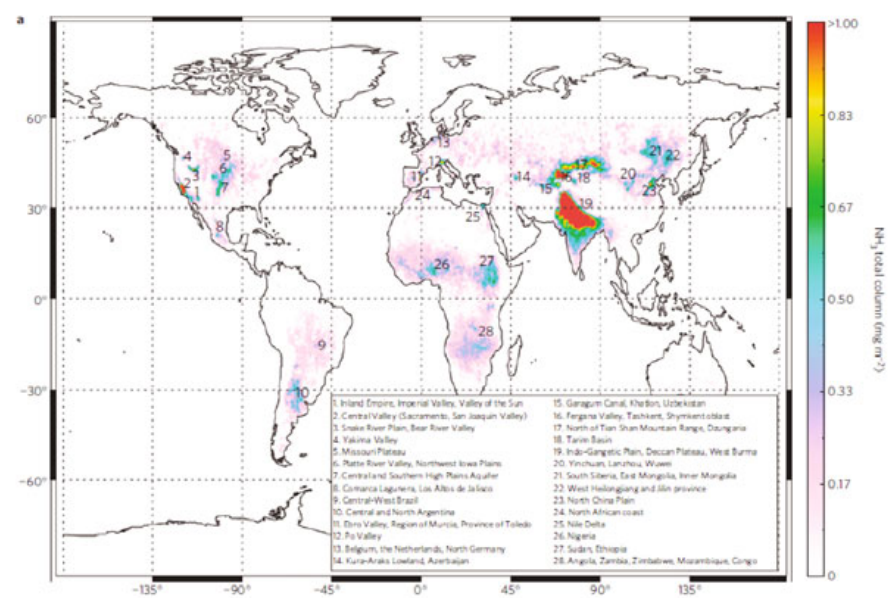

Fig. 6. Yearly average total columns of $\mathrm{NH}_{3}$ in 2008 retrieved from IASI measurements [29].

increase the overall burden of $\mathrm{CO}_{2}$ which has a long atmospheric lifetime and is the most important anthropogenic greenhouse gas.

The signal to noise level, coverage and spatial resolution of the SCIAMACHY NIR measurements are not sufficient to monitor $\mathrm{CO}_{2}$ fluxes on a country or regional level as would be desirable for verification of international treaties on the reduction of greenhouse gas emissions. The problem is not mainly the accuracy (which is very good) but the small size of the anthropogenic signal to be detected on a high background level of $\mathrm{CO}_{2}$. Future instruments with improved signal to noise and much improved spatial resolution and coverage may be able to directly monitor large emission sources such as power plants or cities.

\subsection{IASI detection of tropospheric $\mathrm{NH}_{3}$}

IASI is a Fourier Transform spectrometer covering the spectral range of $645-2.760 \mathrm{~cm}^{-1}$ at $0.5 \mathrm{~cm}^{-1}$ resolution. It provides very good spatial resolution $(12 \mathrm{~km}$ diameter) and near global coverage twice per day. Among many other species, ammonia $\left(\mathrm{NH}_{3}\right)$ can be detected in the spectra around $950 \mathrm{~cm}^{-1}$ [29]. The resulting global map for 2008 is shown in Fig. 6 and highlights many regions of intense 
agriculture, in particular in valley regions where stagnant conditions prevail, but also some areas with strong biomass burning in 2008.

This agrees with expectations as ammonia is mainly released as result of fertilisation which has increased significantly over the last decades, a trend that is expected to continue as an increasing human population has to be fed.

Comparison of the IASI measurements with model results shows overall good agreement but clear indication for model underestimation of $\mathrm{NH}_{3}$ emissions in particular in Central Asia. In some areas, the satellite observations are also lower than model data and in-situ measurements which can be explained by the small sensitivity of thermal infrared measurements to the lowest part of the troposphere in cases of low thermal contrast. Future instrumentation with better spectral resolution could improve the sensitivity for $\mathrm{NH}_{3}$ and in combination with $\mathrm{UV} /$ vis observations of $\mathrm{NO}_{2}$ and potentially also $\mathrm{HONO}$ could provide detailed information on the nitrogen cycle.

\section{Conclusions and outlook}

Satellite observations provide the only way to monitor tropospheric composition on a global scale. The distributions of many different trace gases can now be retrieved from space observations using wavelengths from the UV to the IR. The spectral range selected determines the vertical resolution and sensitivity of the measurements, with thermal IR observations providing the largest list of detectable species and intrinsic vertical resolution while measurements utilising UV and visible wavelengths have sensitivity down to the surface.

Current nadir viewing missions include the GOME, SCIAMACHY, OMI, and GOME-2 instruments in the UV/visible/NIR, and MOPITT, AIRS, TES, and IASI in the thermal IR. In addition, instruments such as OSIRIS, ACE-FTS, and MIPAS can probe the upper troposphere in occultation and limb viewing modes, adding to the overall data set of tropospheric observations. A detailed survey of current capabilities, applications and results can be found in [1].

Improvements for future satellite observations can come both from better instruments and from other platforms used. For UV/vis instruments, better spatial resolution at similar signal to noise is the main aim which can be achieved by larger detectors, larger optics, improved throughput, and full bandwidth data downlink. For IR instruments, better signal to noise can further improve data quality and higher spectral resolution can add more vertical information.

A major step forward would come from the use of geostationary platforms or a constellation of identical satellites in sun-synchronous orbits. Both options would provide measurements at different local times which are needed to a) characterise the diurnal variation of trace gases in the troposphere, b) better constrain models of tropospheric chemistry, c) move towards air quality applications and d) reduce the number of cloud contaminated measurements.

Active systems such as Lidar could play an important role in the future if systems can be built that are sensitive to tropospheric absorbers such as $\mathrm{H}_{2} \mathrm{O}, \mathrm{NO}_{2}$ or $\mathrm{O}_{3}$. Results from the CALIOP instrument on CALIPSO [30] demonstrate the value of aerosol measurements taken at high vertical resolution and similar data for trace species would be of great value. If such Lidars can be built to be stable and sensitive enough to be operated from satellite will have to be seen in the coming years. The poor spatial coverage of these measurements will always necessitate parallel observations using instruments with better spatial coverage.

Parts of this work were funded by the University of Bremen and by the European Union through the ACCENT and CITYZEN projects.

\section{References}

1. Burrows, J.P., Borrell, P., Platt, U. eds, The Remote Sensing of Tropospheric Composition from Space (Springer-Verlag Berlin Heidelberg, 2010)

2. Wagner, T., et al., Journal of Optics a-Pure and Applied Optics 10(10) (2008) 
3. Martin, R.V., Atmospheric Environment 42(34) (2008)

4. Heath, D.F., C.L. Mateer, and A.J. Krueger, Pure and Applied Geophysics 106(5-7) (1973)

5. Fishman, J., et al., Science 252(5013) (1991)

6. Krueger, A.J., Science 220(4604) (1983)

7. Eisinger, M. and J.P. Burrows, Geophysical Research Letters 25(22) (1998)

8. Richter, A., et al., Geophysical Research Letters 25(14) (1998)

9. Palmer, P.I., et al., Journal of Geophysical Research-Atmospheres 106(D13) (2001)

10. Richter, A., et al., Nature 437(7055) (2005)

11. Wittrock, F., et al., Geophysical Research Letters 33(16) (2006)

12. Schönhardt, A., et al., Atmospheric Chemistry and Physics 8(3) (2008)

13. Buchwitz, M., et al., Atmospheric Chemistry and Physics 7(9) (2007)

14. Buchwitz, M., et al., Atmospheric Chemistry and Physics 7(16) (2007)

15. Frankenberg, C., et al., Science 308(5724) (2005)

16. Deeter, M.N., et al., Journal of Geophysical Research-Atmospheres 114 (2009)

17. Aumann, H.H., et al., Ieee Transactions on Geoscience and Remote Sensing 41(2) (2003)

18. Beer, R., T.A. Glavich, and D.M. Rider, Applied Optics 40(15) (2001)

19. Clerbaux, C., et al., Atmospheric Chemistry and Physics 9(16) (2009)

20. Clerbaux, C., et al., Atmospheric Chemistry and Physics 3 (2003)

21. Fischer, H., et al., Atmospheric Chemistry and Physics 8(8) (2008)

22. Yang, K., et al., Geophysical Research Letters 36 (2009)

23. Beirle, S., et al., Atmospheric Chemistry and Physics 9(3) (2009)

24. Boersma, K.F., et al., Journal of Geophysical Research-Atmospheres 113(D16) (2008)

25. Carn, S.A., et al., Geophysical Research Letters 34(9) (2007)

26. Vrekoussis, M., et al., Atmos. Chem. Phys. Discuss. 10(8) (2009)

27. Stavrakou, T., et al., Atmospheric Chemistry and Physics 9(21) (2009)

28. Sinreich, R., et al., Atmos. Chem. Phys. Discuss. 10(6) (2009)

29. Clarisse, L., et al., Nature Geoscience 2(7) (2009)

30. Winker, D.M., W.H. Hunt, and M.J. McGill, Geophysical Research Letters 34(19) (2007) 